\title{
Joint T1 and Brain Fiber Log-Demons Registration Using Currents to Model Geometry
}

\author{
Viviana Siless ${ }^{1,2}$, Joan Glaunès ${ }^{3}$, Pamela Guevara ${ }^{4}$, Jean-François Mangin ${ }^{2}$, \\ Cyril Poupon ${ }^{2}$, Denis Le Bihan ${ }^{2}$, Bertrand Thirion ${ }^{1,2}$, and Pierre Fillard ${ }^{1,2}$ \\ 1 Parietal Team, INRIA Saclay-Île-de-France, Saclay, France \\ viviana.siless@inria.fr \\ http://parietal.saclay.inria.fr \\ 2 CEA, DSV, I ${ }^{2}$ BM, Neurospin bât 145, 91191 Gif-Sur-Yvette, France \\ 3 MAP5, CNRS UMR 8145, Université Paris Descartes, 75006 Paris, France \\ ${ }^{4}$ University of Concepción, Concepción, Chile
}

\begin{abstract}
We present an extension of the diffeomorphic Geometric Demons algorithm which combines the iconic registration with geometric constraints. Our algorithm works in the log-domain space, so that one can efficiently compute the deformation field of the geometry. We represent the shape of objects of interest in the space of currents which is sensitive to both location and geometric structure of objects. Currents provides a distance between geometric structures that can be defined without specifying explicit point-to-point correspondences. We demonstrate this framework by registering simultaneously $T_{1}$ images and 65 fiber bundles consistently extracted in 12 subjects and compare it against non-linear $T_{1}$, tensor, and multi-modal $T_{1}+$ Fractional Anisotropy (FA) registration algorithms. Results show the superiority of the Log-domain Geometric Demons over their purely iconic counterparts.
\end{abstract}

Keywords: Registration, neural fibers, diffeomorphism, Demons Algorithm, intensity-base registration, tensor-base registration, log-domain.

\section{Introduction}

Non-linear image registration is one of the most challenging tasks in medical image analysis. For inter-individual comparison, registration should align images as well as cortical and internal structures such as sulcal lines and fibers. Non-linear registration algorithms can be categorized into iconic, geometric and hybrid.

Iconic, or image-based registration [13310] finds a voxel-wise mapping between a source and a target image. Schematically, iconic registration is mainly driven by the contours of the image, and without prior knowledge, it is difficult to coregister regions with little contrast. For instance, brain white matter appears uniformly white in $T_{1}$ images, giving no relevant information to the iconic registration, while it is composed of neural fibers connecting cortical areas. Diffusion Tensor Imaging (DTI) can be used to reveal the microscopic structure of the white matter. Tensor-based registration was recently proposed to improve 
white matter alignment [17/5]. However, misregistration may persist in regions where the tensor field appears uniform, as shown in [4].

Geometric registration specifically targets the alignment of Structures of Interest (SOI), such as in [16] for cortical surfaces, or [4] for fiber bundles. While those clearly improve SOI registration, they are in general not suitable for aligning other structures than those used specifically during registration.

Hybrid techniques propose to jointly consider SOI and images during registration. For instance, 18 used the mathematical framework of measures and currents to simultaneously register images and geometric descriptors, while [12 proposed a Markovian solution to the same problem.

We present an hybrid registration algorithm based on the efficient framework of Demons, where we combine iconic and geometric registration. The geometry is represented in the space of currents which provides a metric sensitive to shape.

The rest of the paper is organized as follows. First, we propose a mathematically sound extension of the Geometric Demons(GD), the Log-domain Geometric Demons (LGD), that relies in the log-domain framework for computation and handles geometric constraints in the space of currents. Then, we evaluate the LGD with bundles constraints on a dataset of 12 subjects and compare them with a scalar [14, a tensor [15], and Ants [2] a multi-modal registration.

\section{The Log-Domain Geometric Demons}

\subsection{The Diffeomorphic Demons}

In image registration we search for a displacement field $s$ between a fixed $F$ and moving $M$ image, that maps as accurately as possible corresponding structures in both images. Ideally the displacement field $s$ minimizes the distance between the fixed and the moving image, while holding some properties such as being diffeomorphic. In the Demons framework [13] a correspondence field $c$ was introduced to make the minimization of the functional energy tractable: $E(c, s)=\frac{1}{\sigma_{i}^{2}} \operatorname{Sim}(F, M \circ c)+\frac{1}{\sigma_{x}^{2}} \operatorname{dist}(s, c)^{2}+\frac{1}{\sigma_{T}^{2}} \operatorname{Reg}(s)$ where Sim is a similarity measure between images defined by the sum of square differences (SSD) and Reg a regularization term chosen to be an harmonic energy. The amount of regularization is controlled with $\sigma_{T}$ while $\sigma_{i}$ accounts for the image noise. The term dist $(s, c)^{2}$ imposes the displacement field $s$ to be close to the correspondence field $c . \sigma_{x}$ weights the spatial uncertainty on the deformation. The energy minimization is performed by alternating minimization w.r.t. $c$ and $s$. In 14], small deformations parametrized by a dense displacement field $u$ are used: $c \leftarrow s \circ \exp (u), \exp ()$ being the exponential map in the Lie group sense, which ensures that the result is diffeomorphic.

\subsection{Geometric Demons}

To incorporate geometric constraints in the Demons framework, in [1] the definition of $c$ was extended to carry information from both image and geometry. 
Let us denote by $\mathcal{G}^{F}$ (resp. $\mathcal{G}^{M}$ ) the fixed (resp. moving) geometric descriptors. Therefore a new energy was defined:

$$
\begin{aligned}
E(c, s)= & \frac{1}{\sigma_{i}^{2}}\left[\operatorname{Sim}_{I}(F, M \circ c)+\operatorname{Sim}_{G}\left(c \star \mathcal{G}^{F}, \mathcal{G}^{M}\right)\right]+ \\
& \frac{1}{\sigma_{x}^{2}} \operatorname{dist}(s, c)^{2}+\frac{1}{\sigma_{T}} \operatorname{Reg}(s),
\end{aligned}
$$

where $\operatorname{Sim}_{I}$ is the image similarity measure, $\operatorname{Sim}_{G}$ the geometry similarity measure, and $c \star \mathcal{G}^{F}$ denotes the action of $c$ on the geometry.

Following [14, $c$ was parametrized by an update field, as the additive combination of an image update field $u_{I}$ and a geometric update field $u_{G}$. Nonintersecting domains were defined, as ideally, one should use $u_{G}$ only where geometric information is relevant and use $u_{I}$ elsewhere. In the case of fibers, the geometric domain will remain within the white matter. Thus, let $\Omega_{G}$ be the definition domain of $u_{G}$ (where geometry is defined), and the definition domain of $u_{I}$ be $\Omega_{I}=\Omega-\Omega_{G}$. Then we can define $c=\exp \left(u_{I}+u_{G}\right)$. The following relationships hold: $c \star \mathcal{G}^{F}=\exp \left(u_{G}\right) \star \mathcal{G}^{F}$ and $M \circ c=M \circ \exp \left(u_{I}\right)$.

Geometric Demons incorporates the following energy to calculate $u_{G}$ :

$$
E_{G}\left(s, u_{G}\right)=\frac{1}{\sigma_{i}^{2}} \operatorname{Sim}_{G}\left(s \circ \exp \left(u_{G}\right) \star \mathcal{G}^{F}, \mathcal{G}^{M}\right)+\frac{1}{\sigma_{x}^{2}} \int_{\Omega_{G}}\left\|u_{G}\right\|^{2},
$$

Being $s$ the deformation field from $F$ to $M$. Thus, the inverse of the $s$ gives the geometric deformation. In section 2.3 we show an efficient approximation for obtaining the geometric deformation in the log-domain space.

\subsection{Log-Domain Geometric Demons}

The log-domain demons avoids the inversion of the deformation field by redefining $s$ with the exponential map: $s=\exp (v)$. Then $s \circ \exp (u)=\exp (v) \circ \exp (u)$ and the Baker-Campbell-Hausdorff(BCH) formula yields $\log (\exp (v) \circ \exp (u)) \approx$ $v+u+1 / 2[v, u]+1 / 12[v,[v, u]]+\ldots$ where $[v, u]$ is the Lie bracket.

Then, the LogGeometric Demons algorithm is defined as follows:

1. Choose a starting spatial transformation $s=\exp (v)$

2. Given $s, u_{I}$, compute the update field $u_{I}$ as in 14

3. Given $s, u_{G}$, compute the update field $u_{G}$ by minimizing Eq. (2)

4. Let $u \leftarrow s \circ \exp \left(u_{I}+u_{G}\right)$

5. $v \leftarrow \log (\exp (v) \circ \exp (u))$ using $\mathrm{BCH}$ approximation and $\exp (u)$ is efficiently computed with a few compositions, look 14 for further details.

6. let $v \leftarrow K_{\text {diff }} \star v$ where $K_{\text {diff }}$ is a Gaussian convolution kernel

7. $s=\exp (v)$ and $s^{-1}=\exp (-v)$

8. Go to 2. until convergence

With this new definition we can efficiently compute the geometric deformation. 
Calculation of $\boldsymbol{u}_{\boldsymbol{G}}$ in the Space of Currents. In GD the closest point distance was proposed as it does not need explicit correspondences between points and it can be a good approach for comparing single fiber bundles representatives as in 11. In contrast, by representing geometry in the space of currents, we have a pose and shape-sensitive measure which permits to define a distance between bundles containing different number of fibers.

Let $\mathcal{G}$ be a set of continuous curves. We define the sequence of discretized points in $\mathcal{G}$ as $\mathcal{G}=\left(x_{1}, \ldots, x_{N}\right), N$ being the number of points. We can associate to this sequence a specific measure given by the vector valued Diracs: $\mu_{\mathcal{G}}=$ $\sum_{i=1}^{n-1} \tau_{\mathcal{G}, i} \delta_{c_{\mathcal{G}}, i}$ where $c_{\mathcal{G}, i}=\left(x_{i}+x_{i+1}\right) / 2$ (center point) and $\tau_{\mathcal{G}, i}=x_{i+1}-x_{i}$ (tangent vector) if $x_{i}$ and $x_{i+1}$ belong to the same curve, otherwise $\tau_{\mathcal{G}, i}=0$.

Following [6], let $W$ be a reproducible kernel Hilbert space (r.k.h.s) of vector fields with kernel $K_{\beta}^{W}$ isotropic and Gaussian of size $\beta$ : vector fields in $W$ are convolutions between any square-integrable vector fields and the convolution square root of the kernel. Then, the vector space of currents is a dense span of the set of all the vector valued Diracs currents $\tau \delta_{c}$ for any $\tau, c \in \mathbb{R}^{3}$. A Dirac current may be seen as an oriented segment entirely concentrated at point $c$. The scalar product between two sums of vector valued Diracs expresses conveniently in terms of the kernel $K_{\beta}^{W}$ :

$$
\left\langle\mu, \mu^{\prime}\right\rangle=\left\langle\sum_{i=1}^{N} \tau_{i} \delta_{c_{i}}, \sum_{j=1}^{M} \tau_{j}^{\prime} \delta_{c^{\prime}{ }_{j}}\right\rangle=\sum_{i=1}^{N} \sum_{j=1}^{M} K_{\beta}^{W}\left(c_{i}, c_{j}^{\prime}\right) \tau_{i} . \tau_{j}^{\prime}
$$

Having a fixed and a moving geometric descriptor $\mathcal{G}^{F}=\left(x_{1}, \ldots, x_{N}\right)$ and $\mathcal{G}^{M}=$ $\left(y_{1}, \ldots, y_{M}\right) N, M$ being the number of points, the distance is defined as follows:

$$
d^{2}\left(\mathcal{G}^{F}, \mathcal{G}^{M}\right)=\left\|\mathcal{G}^{F}-\mathcal{G}^{M}\right\|_{W^{*}}^{2}=\left\|\mathcal{G}^{F}\right\|_{W^{*}}^{2}+\left\|\mathcal{G}^{M}\right\|_{W^{*}}^{2}-2\left\langle\mathcal{G}^{F}, \mathcal{G}^{M}\right\rangle_{W^{*}}
$$

The distance measures geometrical differences both in pose and shape. With $\beta$ we define the kernel size, and points at distances much larger than $\beta$ have a large distance disregarding the shape. Also, when distances are much smaller than $\beta$, they are taken as noise, and thanks to the smoothing effect of the kernel they are not taken into account. So the distance captures first misalignment and then shape dissimilarities until a noise level quantified by $\beta$ is reached.

Let us define the action of the correspondence field $c$ on $\mathcal{G}$ as: $c \star \mathcal{G}=\{s \circ$ $\left.\exp \left(u_{G}\right)\left(x_{i}\right)\right\}_{i \in[1, N]} \approx\left\{s\left(x_{i}\right)+u_{G}\left(x_{i}\right)\right\}_{i \in[1, N]}$. Since we are dealing with discrete points, we choose to parametrize the dense update field $u_{G}$ by a finite set of vectors $u_{G, i}$ using radial basis function interpolation: $u_{G}(x)=\sum_{i=1}^{N} h(\| x-$ $\left.x_{i} \|\right) \lambda_{i}$. When $h(x)=e^{-(r)^{2}}, \lambda_{i}$ are calculated such that $u_{G}\left(x_{i}\right)=u_{G, i} \forall i$. Let us define the matrix $A$ such that $[A]_{i, j}=h\left(\left\|x_{i}-x_{j}\right\|\right)\left([A]_{i, j}\right.$ denotes the $(i, j)$ entry of $A), H(x)$ the vector such that $[H(x)]_{i}=h\left(\left\|x-x_{i}\right\|\right)$ and $U=\left[u_{G, 1}, \ldots, u_{G, N}\right]$. We can write: $u_{G}(x)=H(x) A^{-1} U$. Solving $\nabla E_{G}\left(s, u_{G}\right)=0$ w.r.t. $u_{G}$ narrows down to optimization for the $u_{G, i}, \forall i$. After differentiation, we obtain:

$$
u_{G, i}=\frac{\nabla_{u_{G, i}}\left\|c \star \mathcal{G}^{F}-\mathcal{G}^{M}\right\|_{W^{*}}^{2}}{1+\frac{\sigma_{i}^{2}}{\sigma_{x}^{2}}\left[H\left(s\left(x_{i}\right)\right) A^{-1}\right]_{i}}
$$


Defining $\Omega_{G}$ for Bundles. Since we want fibers to influence the deformation near the definition domain, we define the domain as the union of $\gamma$-radius balls $B$ centered at each coordinate $x_{i}$. We control the influence by varying $\gamma$ and thus, dilating the domain. We define a binary map $\Omega_{G}^{\gamma}=\bigcup_{i=1}^{N} B\left(x_{i}, \gamma\right)$. The domain of the image correspondence field is the complementary of $\Omega_{G}^{\gamma}: \Omega_{I}^{\gamma}=\Omega \backslash \Omega_{G}^{\gamma}$.

\section{Joint $T_{1}$ MRI and Brain Bundle Registration}

\subsection{Data Description}

We used the NMR database of 12 healthy volunteers scanned with $T_{1}(256 \times 256 \times$ $124, .9375 \times .9375 \times 1.2 \mathrm{~mm})$ and DW-MRI $(128 \times 128 \times 60,1.875 \times 1.875 \times 2 \mathrm{~mm})$ 9. 200 encoding gradients were used for the diffusion sequence. Using [7, we obtained corresponding fiber bundles between several subjects. 100 bundles were consistently identified in all subjects. The longest 65 bundles distributed in both hemispheres were retained for the experiments. For each subject we obtained the linear transformation from $B_{0}$ to $T_{1}$ to align bundles with $T_{1}$ images.

\section{$3.2 \quad$ Experiments}

Two experiments were conducted. First, we exhaustively analyze the parameter $\gamma$ in $\omega_{G}^{\gamma}$ defined in Sec. 2.3 to understand its effect on registration accuracy. Second, we compared the performance between Symmetric Log Domain Demons (SLDD), the Symmetric Tensor Demons (STD) and Ants. The inverted deformation field was applied to the fibers to display the registration. Each algorithm was tested on the 3 -steps multi-scale approach with 15, 10 and 5 iterations at each scale (from small to large). We set the currents kernel size $\beta$ by using a robust estimator of the maximum distance between bundles thresholded at $20 \mathrm{~mm}$.

Influence of $\gamma$. In the first experiment, the 11 subjects were registered onto one, arbitrary chosen as the target subject. We varied $\gamma$ from 0 to 4.5, where $\gamma$ is scaled by the smallest voxel size. We divided our bundles in 5 sets (13 bundles each, with bundles of $\sim 3$ fibers, each of 21 points), and used jointly 4 to train, and the left one to test. The following results show the average of the 5 possible permutations of choosing the test set. We show results over training set (a), test set (b), and the image (c) for the increasing values of $\gamma$ in Fig. 1. As expected, fiber matching improves as $\gamma$ increases(a) at the expense of image alignment(c). Indeed, when fibers have a large influence on their neighborhood, image-driven forces are discarded, leading to poor image registration. Also, comparing (a) and (b) we note that $\gamma=4.5$ is overfitting the fibers, misleading the overall registration. $\gamma=3.0$ largely improves fiber alignment, while keeping a good match between images. In the sequel, a $\gamma=3.0$ will be used. In some cases image matching is slightly improved when using $\gamma=1.5$ pointing out that geometry may indeed help image registration to avoid local minima. 


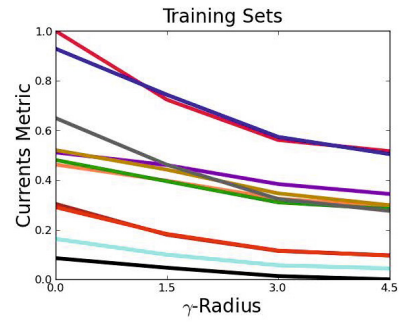

(a)

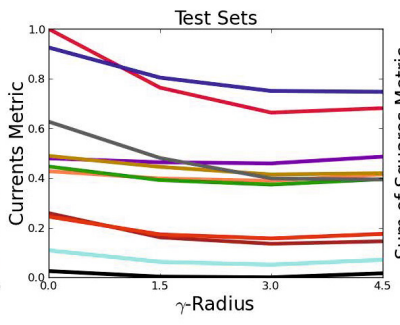

(b)

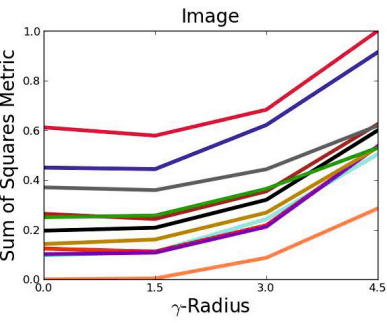

(c)

Fig. 1. Influence of $\gamma$. Similarity measure average of the 5 training set permutations with varying $\gamma$. Different color curves represent the 11 subjects.

Comparison with SLDD, STD and ANTS. For this experiment we register all subjects to each subject for each permutation of the training set. For SLDD, we registered $11 T_{1}$ images onto the 12 permuted target and applied the inverted deformation field to the bundles. For STD, we extracted tensors using [5] and registered each of them onto the 12 permuted target tensor image. Then, inverted deformation fields were applied to each subject's fibers in the DWI space. Finally, the linear transformation calculated between the target $B_{0}$ and $T_{1}$ images was used to carry fibers to the $T_{1}$ space. For Ants, we extracted the FA from the tensors obtained using [5], and aligned them to their $T_{1}$ image. We use the cross correlation setting with weighting equally image and FA. Then affine and nonrigid resulting transformation were applied to images, while the inverse of the non-rigid and the affine were applied to the fibers. We show the average metric over training sets (i), test sets $(\mathrm{j})$ and image $(\mathrm{k})$ of registering all subjects to each one with the methods mentioned above in Fig. 2 .

As expected, LGD further improved fiber registration in (i) compared to the other algorithms. However, the training set contains the fibers used during registration; we explicitly optimize a metric evaluated on those fibers. Analyzing the results over the test set in $(\mathrm{j})$ we see a similar performance compared to Ants, and STD, which is remarkable as STD is using information from tensors over the whole dense grid, and Ants does a cross correlation between the whole $F A$ grid and $T_{1}$. By contrast, in LGD the deformation field was obtained using only sparse information coming from selected fibers, which are not defined in the regions tested in $(\mathrm{j})$. Therefore having a similar performance is very promising. We can also see in $(\mathrm{k})$ the image registration for STD was extremely poor. We time all algorithms with an Intel Xeon 8proc. $2.53 \mathrm{GHz}, 11.8 \mathrm{~Gb}$ and obtained: $\mathrm{SLDD}=19.61 \mathrm{~min}, \mathrm{STD}=10.75 \mathrm{~min}, \mathrm{Ants}=25.63 \mathrm{~min}$, and $\mathrm{LGD}=12.51 \mathrm{~min}$.

\section{Discussion}

We compared our algorithm against a scalar (SLDD), a tensor (STD), and a multi-modal (Ants) registration. Results show that bundle alignment was highly improved comparing to other algorithms. We get accurate results even for testing 
set fibers where no information was used from the support regions of the those fibers, and STD and Ants were using information from the whole grid. This shows that a small set of fibers might be sufficient for a proper registration of the white matter across subjects. Moreover, while fiber alignment is improved, the efficiency of the image alignment is maintained. When evaluating the algorithm for the different $\gamma$ values, we could see that $\gamma=1.5$ better registers missing structures than $\gamma=0$ and than SLDD. However, we believe there is a trade-off to make between image and fiber alignment, and $\gamma=3$ notably improved fiber alignment while still obtaining good image registration results. By using labeled

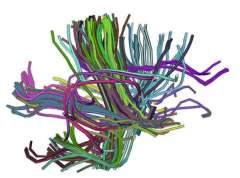

(d) Original

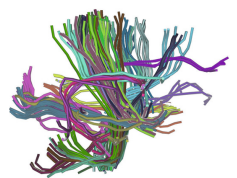

(e) LGD

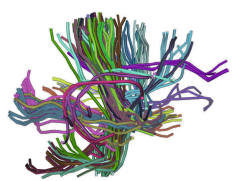

(f) SLDD

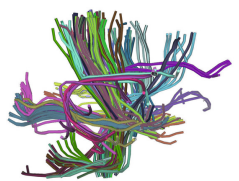

(g) STD

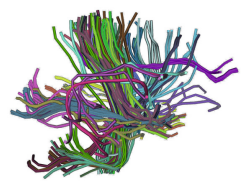

(h) Ants

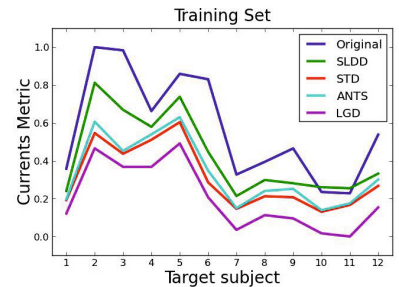

(i)

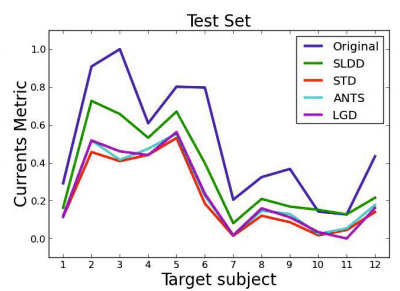

(j)

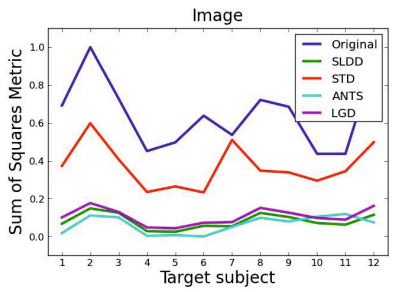

$(\mathrm{k})$

Fig. 2. Comparison of SLDD, STD, Ants and LGD Top: Target fibers overlapped with registered fibers from an arbitrary chosen registration. 29 fibers were arbitrary preselected for clarity. Corresponding fibers in subjects share colors. Bottom: Average of the metrics obtained from registering 11 subjects to the target subject.

bundles instead of purely tensor information, we add relevant features that were previously extracted as prior such as region connection. Nevertheless, the efficacy of trusting bundles is open to discussion, and bundles classification is an active topic in research, so we believe this information should not be discarded.

\section{Conclusion}

We extended the GD algorithm to the log-domain space, and combined it with currents to compare our geometric structures and respect their shape. Our algorithm perfomed similarly as other competitive algorithms on image and showed a large improvement in fiber alignment. A unique mapping for images and geometric structures is obtained, giving a consistent framework for analyzing and comparing results between voxel-based morphometry and shape of SOI.

Having disjoint domains for geometry and iconic may potentially limit the incorporation of new geometric structures such as sulcal lines or features. This 
choice was done to guarantee a closed-form solution when zeroing out the gradient of the criterion. Moreover, both update fields are eventually combined and smoothed out, lowering the effect of the disjoint domains. Future work will further address the remaining mathematical issues to allow overlapping domains.

Based on the efficiency of the algorithm, it would be interesting to combine it with the clustering of fibers: increased fiber registration can help clustering algorithms, which can in turn guide the registration.

Acknowledgements. This work was supported by the INRIA CapNeonates Equipe Associée.

\section{References}

1. Auzias, G., et al.: Diffeomorphic brain registration under exhaustive sulcal constraints. IEEE Trans. Med. Imaging (January 2011)

2. Avants, B.B., et al.: A reproducible evaluation of ants similarity metric performance in brain image registration. NeuroImage 54(3), 2033-2044 (2011)

3. Beg, M.F., et al.: Computing large deformation metric mappings via geodesic flows of diffeomorphisms. IJCV 61(2), 139-157 (2005)

4. Durrleman, S., et al.: Registration, atlas estimation and variability analysis of white matter fiber bundles modeled as currents. NeuroImage 55(3), 1073-1090 (2011)

5. Fillard, P., et al.: Clinical DT-MRI estimation, smoothing and fiber tracking with log-Euclidean metrics. IEEE Trans. Med. Imaging 26(11), 1472-1482 (2007)

6. Glaunès, J., et al.: Large deformation diffeomorphic metric curve mapping. IJCV 80, 317-336 (2008), 10.1007/s11263-008-0141-9

7. Guevara, P., et al.: Robust clustering of massive tractography datasets. Neuroimage 54(3), 1975-1993 (2011)

8. Ha, L., Prastawa, M., Gerig, G., Gilmore, J.H., Silva, C.T., Joshi, S.: Image Registration Driven by Combined Probabilistic and Geometric Descriptors. In: Jiang, T., Navab, N., Pluim, J.P.W., Viergever, M.A. (eds.) MICCAI 2010, Part II. LNCS, vol. 6362, pp. 602-609. Springer, Heidelberg (2010)

9. Poupon, C., et al.: A database dedicated to anatomo-functional study of human brain connectivity. In: 12th HBM Neuroimage, Florence, Italie, vol. 646 (2006)

10. Rueckert, D., Aljabar, P., Heckemann, R.A., Hajnal, J.V., Hammers, A.: Diffeomorphic Registration Using B-Splines. In: Larsen, R., Nielsen, M., Sporring, J. (eds.) MICCAI 2006. LNCS, vol. 4191, pp. 702-709. Springer, Heidelberg (2006)

11. Siless, V., Guevara, P., Pennec, X., Fillard, P.: Joint T1 and Brain Fiber Diffeomorphic Registration Using the Demons. In: Liu, T., Shen, D., Ibanez, L., Tao, X. (eds.) MBIA 2011. LNCS, vol. 7012, pp. 10-18. Springer, Heidelberg (2011)

12. Sotiras, A., Ou, Y., Glocker, B., Davatzikos, C., Paragios, N.: Simultaneous Geometric - Iconic Registration. In: Jiang, T., Navab, N., Pluim, J.P.W., Viergever, M.A. (eds.) MICCAI 2010, Part II. LNCS, vol. 6362, pp. 676-683. Springer, Heidelberg (2010)

13. Thirion, J.P.: Image matching as a diffusion process: an analogy with Maxwell's demons. Medical Image Analysis 2(3), 243-260 (1998)

14. Vercauteren, T., Pennec, X., Perchant, A., Ayache, N.: Symmetric Log-Domain Diffeomorphic Registration: A Demons-Based Approach. In: Metaxas, D., Axel, L., Fichtinger, G., Székely, G. (eds.) MICCAI 2008, Part I. LNCS, vol. 5241, pp. 754-761. Springer, Heidelberg (2008) 
15. Yeo, B., et al.: Dt-refind: Diffusion tensor registration with exact finite-strain differential. IEEE Trans. Med. Imaging 28(12), 1914-1928 (2009)

16. Yeo, B., et al.: Spherical demons: Fast diffeomorphic landmark-free surface registration. IEEE Trans. Med. Imaging 29(3), 650-668 (2010)

17. Zhang, H., et al.: Deformable registration of diffusion tensor $\mathrm{mr}$ images with explicit orientation optimization. Medical Image Analysis 10(5), 764-785 (2006) 